\title{
Regeneration of Autotransplanted Avascular Lymph Nodes in the Rat Is Improved by Platelet-Rich Plasma
}

\author{
Catarina Hadamitzky ${ }^{\mathrm{a}}$ Katrin S. Blum ${ }^{\mathrm{b}}$ Reinhard Pabst ${ }^{\mathrm{a}}$ \\ anstitute for Functional and Applied Anatomy, and ${ }^{\mathrm{b} C l i n i c}$ for Diagnostic Radiology, Hannover Medical School, \\ Hannover, Germany
}

\section{Key Words}

Lymph node transplant - Lymphatic vessel $\cdot$ Platelet-rich plasma $\cdot$ Growth factors $\cdot$ Lymphedema prevention model

\begin{abstract}
The aim of this study was to verify that subcutaneous lymph node transplantation inducing lymphatic regeneration is possible in healthy adult rats, in analogy to results obtained in other species. This rat model was used to determine the effects of lymph node fragmentation as well as sheep erythrocytes and platelet-rich plasma injection on the regeneration of the transplanted lymph nodes. The results show for the first time that the rat is an adequate model to study the regeneration of transplanted lymph nodes. Lymph node fragmentation seems to affect transplant regeneration negatively. An immune challenge by injection of sheep erythrocytes in the drainage area of the transplanted lymph nodes does not improve fragment regeneration. However, injection of syngeneic platelet-rich plasma containing several growth factors resulted in an improvement in regeneration. Lymph node fragment regeneration, although still experimental, could be relevant for lymphedema prevention. Acquired lymphedema has a high prevalence in developed countries as a consequence of the removal and/or radiotherapy of tumor-draining lymph nodes in cancer patients. This disease causes lifelong disability due to chronic swelling and increased risk of infections. It currently lacks an effective treatment.

Copyright $\odot 2009$ S. Karger AG, Basel
\end{abstract}

\section{Introduction}

Effective lymphatic transport is influenced by multiple factors including the rate of capillary filtration, muscular activity, breathing movements and body position. The integrity of lymphatic vessels, their muscular walls and valves, as well as of lymph nodes is of major importance in this delicate balance. Lymph nodes do not only exert an immunological function but also selectively reabsorb lymph fluid in their blood capillaries, therefore concentrating the lymph $[1,2]$. A disruption of lymph transport in healthy individuals induces lymphatic regeneration. When regenerative mechanisms are insufficient to respond to the lymphatic load, proteins and fluid accumulate peripherally causing lymphedema.

Acquired lymphedema of the extremities affects a high percentage of patients having undergone oncologic surgery with lymphadenectomy and/or lymphatic radiation. Up to $30 \%$ of breast cancer survivors develop a lymphedema of the ipsilateral arm after these procedures at some point in their lives [3]. Following lymphadenectomy for cervix cancer or melanoma of the extremities, the incidence is about $20 \%$ [3].

Lymphedema is extremely disabling [4] and represents a lifelong condition. Its management currently consists of symptom palliation. Many surgical methods with curative aims have been proposed without ground-breaking results, so that none could implement itself as part of the common treatment.

\section{KARGER}

Fax +41613061234 E-Mail karger@karger.ch www.karger.com
Dr. med. Catarina Hadamitzky

Institute for Functional and Applied Anatomy, Hannover Medical School

Carl-Neuberg-Strasse 1, DE-30625 Hannover (Germany)

Tel. +49511532 6742, Fax +495115322948

E-Mail Hadamitzky.Catarina@MH-Hannover.de 


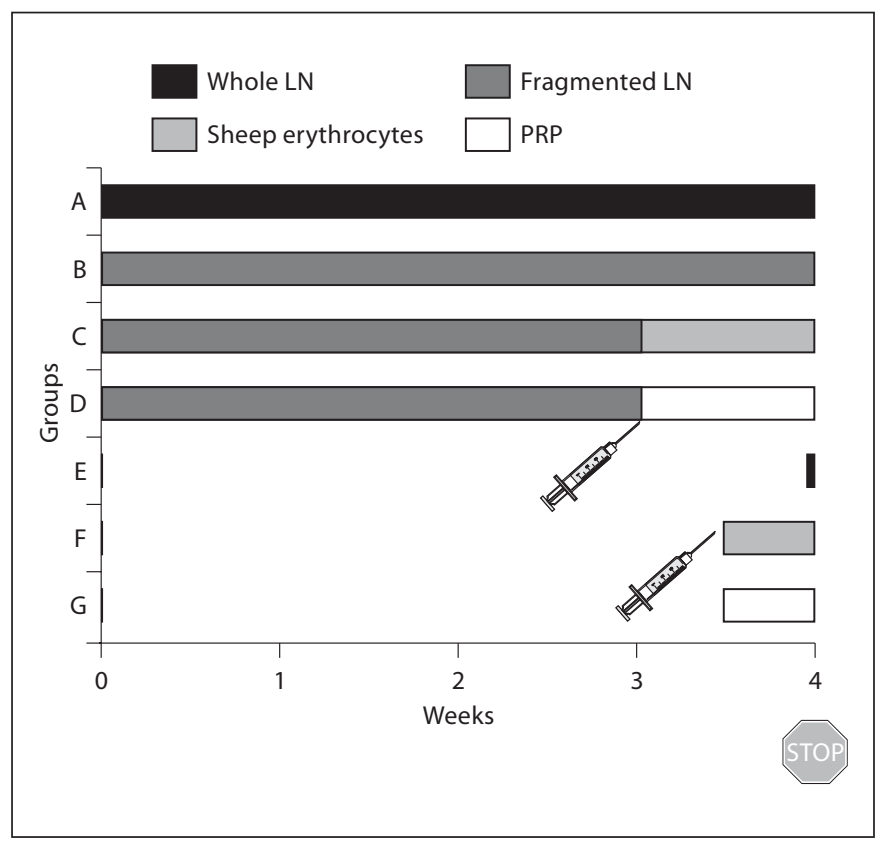

Fig. 1. Therapy and control groups. Groups A-D, 8 rats for each group; groups $\mathrm{E}-\mathrm{G}, 4$ rats for each group. In groups $\mathrm{C}$ and $\mathrm{D}$, postoperative injection was done at 3 weeks. In groups $\mathrm{F}$ and $\mathrm{G}$, injection was performed 3 days before sacrifice, as our kinetic studies showed improved reactivity in this period. $\mathrm{LN}=\mathrm{Lymph}$ nodes.

Research on preventive measures for lymphedema led to the concept of lymph node transplantation [5]. Avascular subcutaneous lymph node transplantation has proven to be possible in the pig [6]. It is documented that these transplants regenerate to functioning lymph nodes and are connected to surrounding lymphatic vessels [58]. Nevertheless, immunohistological characterization of the transplants was limited, as no lymph vessel markers currently exist for swine.

Tammela and colleagues [9] very recently established a mouse model for avascular lymph node transplantation. Their experiments mainly focused on the growth and remodeling of lymphatic capillaries into functional collecting lymphatic vessels, including valve formation and endothelial cell-cell junctions.

The aim of the present study was to develop an experimental model in the rat to ascertain subcutaneous lymph node transplantation in this species. Additionally, the influence of lymph node fragmentation on transplant regeneration was tested. Lymph node fragmentation is important as it may allow multiplication of lymphatic regeneration foci. The effects of sheep erythrocytes as par- ticulate antigens or autologous platelet-rich plasma (PRP) injections after fragment transplantation were also analyzed.

\section{Materials and Methods}

\section{Animals}

The experimental procedure was reviewed and authorized by the local authorities. All operations were performed under full anesthesia with intraperitoneal ketamine $(90 \mathrm{mg} / \mathrm{kg}$; Albrecht $\mathrm{GmbH}$, Aulendorf, Germany) and medetomidine hydrochloride (0.25 mg/kg; Pfizer GmbH, Karlsruhe, Germany). Metamizol was added to the drinking water during the first 2 postoperative days (8 dps/500 ml water; Ratiopharm GmbH, Ulm, Germany).

\section{Study Design}

This study included 44 female Lewis rats (approximately 200 g) divided into therapy and control groups. The therapy group consisted of 32 animals divided into 4 groups of 8 rats each. They were subjected to a bilateral extirpation of the superficial inguinal lymph nodes. An ipsilateral autologous implantation of whole (group A) and fragmented (groups B, C and D) lymph nodes followed. Fragments were approximately $3 \mathrm{~mm}$ in diameter. Lymph nodes were reimplanted in a subcutaneous inguinal pouch behind the caudal nipples, in order to have an anatomical reference for the placement. The pouch and the incision were closed with absorbable subcutaneous sutures (Marlin Violet, Catgut $\mathrm{GmbH}$, Markneukirchen, Germany). In groups A and B, the transplants were left to regenerate without further intervention. In group C, sheep red blood cells $(0.2 \mathrm{ml} / \mathrm{rat}$; Institut Virion/Serion, Würzburg, Germany) were injected intradermally in the lymphatic drainage area of the subcutaneous inguinal pouch 3 weeks after operation. In group D, PRP was injected intradermally 3 weeks after surgery. All rats were euthanized after 1 month. Transplanted lymph nodes were then examined macroand microscopically.

In the control group, 12 animals were divided into 3 groups of 4 rats. No operation was performed. Group E was used as a normal lymph node control. In group $\mathrm{F}$, an intradermal injection of sheep red blood cells was given in the drainage area of the inguinal lymph nodes, and in group G, PRP was injected. Groups F and G were euthanized 3 days after injection, and the lymph nodes analyzed (fig. 1).

\section{Preparation of $P R P$}

Blood was drawn from 8 female Lewis rats via abdominal aorta puncture into heparin tubes (7.5-ml tubes without pellets; Sarstedt AG \& Co. Nümbrecht, Germany). A total of $35 \mathrm{ml}$ blood was collected with a baseline platelet count of $291,666 / \mu 1$. This anticoagulated blood was first submitted to a soft spin of $100 \mathrm{~g}$ for $15 \mathrm{~min}$ at $25^{\circ} \mathrm{C}$ to isolate plasma and platelets in the supernatant. The supernatant was taken with the buffy coat and a fine layer of the upper erythrocytes and submitted to a hard spin of $1,000 \mathrm{~g}$ for $10 \mathrm{~min}$. Platelet-poor supernatant was eliminated until $2 \mathrm{ml}$ PRP remained at the bottom of the tube. The platelet concentration of PRP was $1,545 \times 10^{3} / \mu$ l. Platelets were activated with a $10 \%$ solution of calcium chloride $\left(300 \mu \mathrm{CaCl}_{2} / \mathrm{ml}\right)$ and $300 \mathrm{IU} / \mathrm{ml}$ of bovine thrombin (Merck KGaA, Darmstadt, 
Germany), and this mixture was left to rest for $1 \mathrm{~h}$ [10-12]. Rats were given a short anesthesia with isoflurane gas (Baxter, Unterschleissheim, Germany), and $200 \mu$ l PRP divided into 4 portions of $50 \mu \mathrm{l}$ was injected intradermally in the drainage area of the transplanted lymph nodes of each rat in group D and control group $\mathrm{G}$ (in the medial knee zone and the lateral aspect of the tail base).

\section{Macroscopic Examination}

Functional analysis of lymph drainage was accomplished in therapy groups $\mathrm{B}, \mathrm{C}$ and $\mathrm{D}$ through intradermal injection of small particle blue dye (Patentblau V, Guerbet GmbH, Sulzbach, Germany) in the hind paws under anesthesia. Hereafter, flexion and extension movements of the hind legs were performed for $1 \mathrm{~min}$. The skin was resected, and the connections and staining of the transplanted lymph nodes registered. The animals were then sacrificed by cervical dislocation and the transplant area of the inguinal pouch harvested for histological analysis. If lymph node fragments were not visible in the area of the pouch, additional probes were taken in the original operative field.

\section{Histological Analysis}

Tissue samples were immediately frozen in liquid nitrogen. The sections were stained with IgG against B lymphocytes (BM4013, Acris Antibodies GmbH, Hiddenhausen, Germany), IgG against T lymphocytes (R73, Serotec $\mathrm{GmbH}$, Düsseldorf, Germany), IgG for detection of high endothelial venules (HEVs) by binding to the RECA-1 marker (His 52, MorphoSys AG, Martinsried, Germany), antibodies against dendritic cells and macrophages (MRC OX-6, Serotec), as well as antibodies against lymphatic endothelial cells Proxl and LYVE-1 (ReliaTech GmbH, Braunschweig, Germany). Lymph node structural organization was registered. The transplant was considered organized and regenerated when B lymphocytes were displayed peripherally with follicular distribution, T cells in the central zone of the lymph node and dendritic cells were numerous and distributed uniformly. The presence and distribution of HEVs were analyzed as proof of a connection with the vascular system. Proxl and LYVE-1 patterns were taken as indicators for lymphatic regeneration. Prox1 is present in the cytoplasm of lymphatic endothelial cells only and LYVE-1 receptor in the luminal aspect of lymphatic vessels $[13,14]$.

\section{Results}

\section{Macroscopic Examination}

Intradermal injection of blue dye permitted visualization of superficial abdominal lymphatic vessels in all animals subjected to this procedure $(n=24)$. However, lymph node fragments were stained in only 13 of 48 operation sites (fig. 2). All stained fragments were confirmed by microscopy as being lymph node transplants. In 2 of these animals, the blue stained transplanted fragments were found outside of the original subcutaneous pouch. Thus, fragments were able to migrate out of the pouch despite closure with single stitches. This migra-

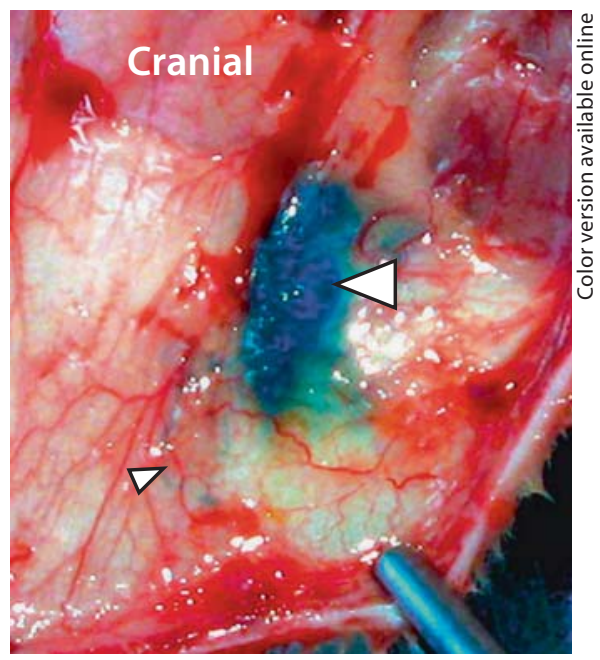

Fig. 2. Regenerated transplanted lymph nodes participate in lymphatic drainage. Blue stained transplanted lymph nodes (big arrowhead) in the inguinal pouch, with afferent lymphatic vessels (small arrowhead). Patent blue dye, exclusively drained by lymphatics, had previously been injected in the peripheral draining area of the nodes. The colors refer to the online version of the figure.

tion did not seem to compromise lymph node regeneration.

\section{Microscopic Examination of Control Groups}

After peripheral administration of sheep red blood cells, lymph nodes of non-operated rats in control group F were harvested. Surprisingly, histological analysis did not show significant differences from control group $\mathrm{E}$ (no intervention).

After administration of syngeneic PRP in group G, lymph node histology strongly contrasted with control group E. Very numerous small secondary follicles were observed in the paracortical region as a clear sign of proliferative reaction.

\section{Microscopic Examination of Therapy Groups}

As previously mentioned, postoperative lymph node dislocation occurred. Therefore, not all tissue samples contained lymph nodes. In the therapy groups $(\mathrm{A}, \mathrm{B}, \mathrm{C}$ and D), lymph nodes (whether necrotic or viable) were detected microscopically at 37 of 64 operation sites (57.8\%). The remaining probes were devoid of transplants. Microscopic analysis of transplanted lymph nodes could be performed at 11 operation sites $(68.8 \%)$ in group $\mathrm{A}$ as well as in group B, at 8 sites in group C (50\%) and at 7 sites in group D (43.8\%). 


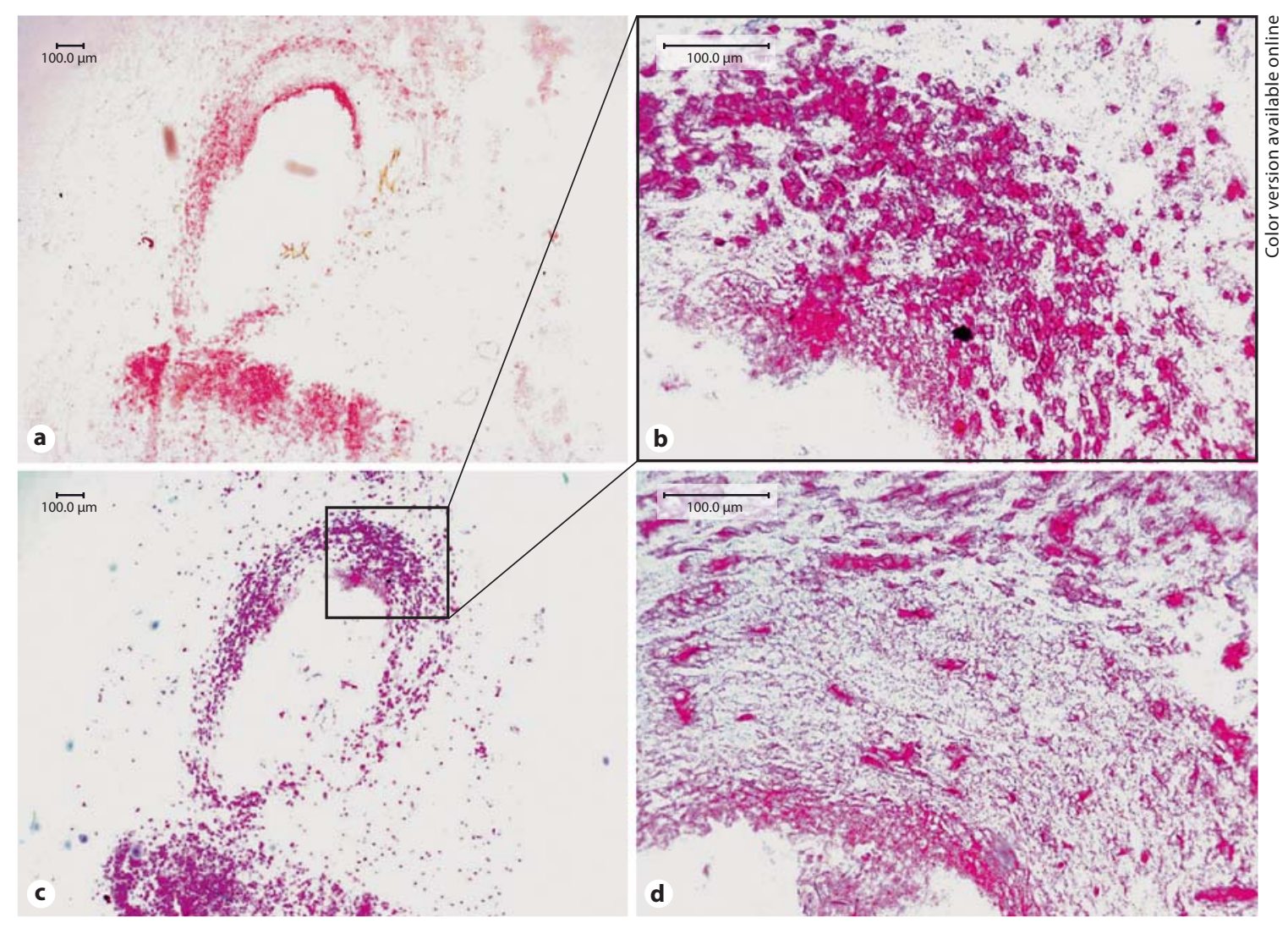

Fig. 3. Necrotic lymph node fragment 4 weeks after autologous transplantation (group B). Cryosections were analyzed by immunohistological staining against B lymphocytes (a), T lymphocytes (b, c) and HEV RECA-1 marker (d).

\section{B Lymphocytes, T Lymphocytes, Dendritic Cells and} HEVs

According to the B- and T-cell patterns, the dendritic cell distribution and the presence of HEVs, lymph node transplants were registered as necrotic (fig. 3 ) or as organized and regenerated (fig. 4). Necrotic lymph node tissue was characterized by a central necrosis surrounded by a T-lymphocyte corona, absence of B cells and a low dendritic cell population. Organized lymph node fragments presented peripheral B-cell follicles, a central T-lymphocyte area with a background rich in dendritic cells and an even distribution of HEVs, as seen in lymph nodes of control group $\mathrm{E}$ (no intervention).

\section{Prox 1 and LYVE-1}

In all viable fragments, Prox 1 and LYVE-1 staining appeared reinforced along the subcapsular and medullary sinuses (fig. 5). This pattern was consistent with the one observed in control group $\mathrm{E}$ and was not present in necrotic lymph nodes.

In group $A, 9$ of the whole lymph node transplants were organized (81.8\%), 1 was necrotic and in 1 groin with 2 transplanted lymph nodes, 1 was functional and 1 necrotic. In group B, 7 (63.6\%) of the fragmented lymph node clusters were functional, and 4 (36.4\%) presented adjacent regenerated and necrotic fragments. In group C, after stimulation with sheep erythrocytes, 4 clusters were fully regenerated (50\%), 3 were necrotic (37.5\%) and 1 presented regenerated and necrotic fragments. Interestingly, in group D, after administration of PRP, all 7 probes showed regenerated lymph node fragments (fig. 6). 


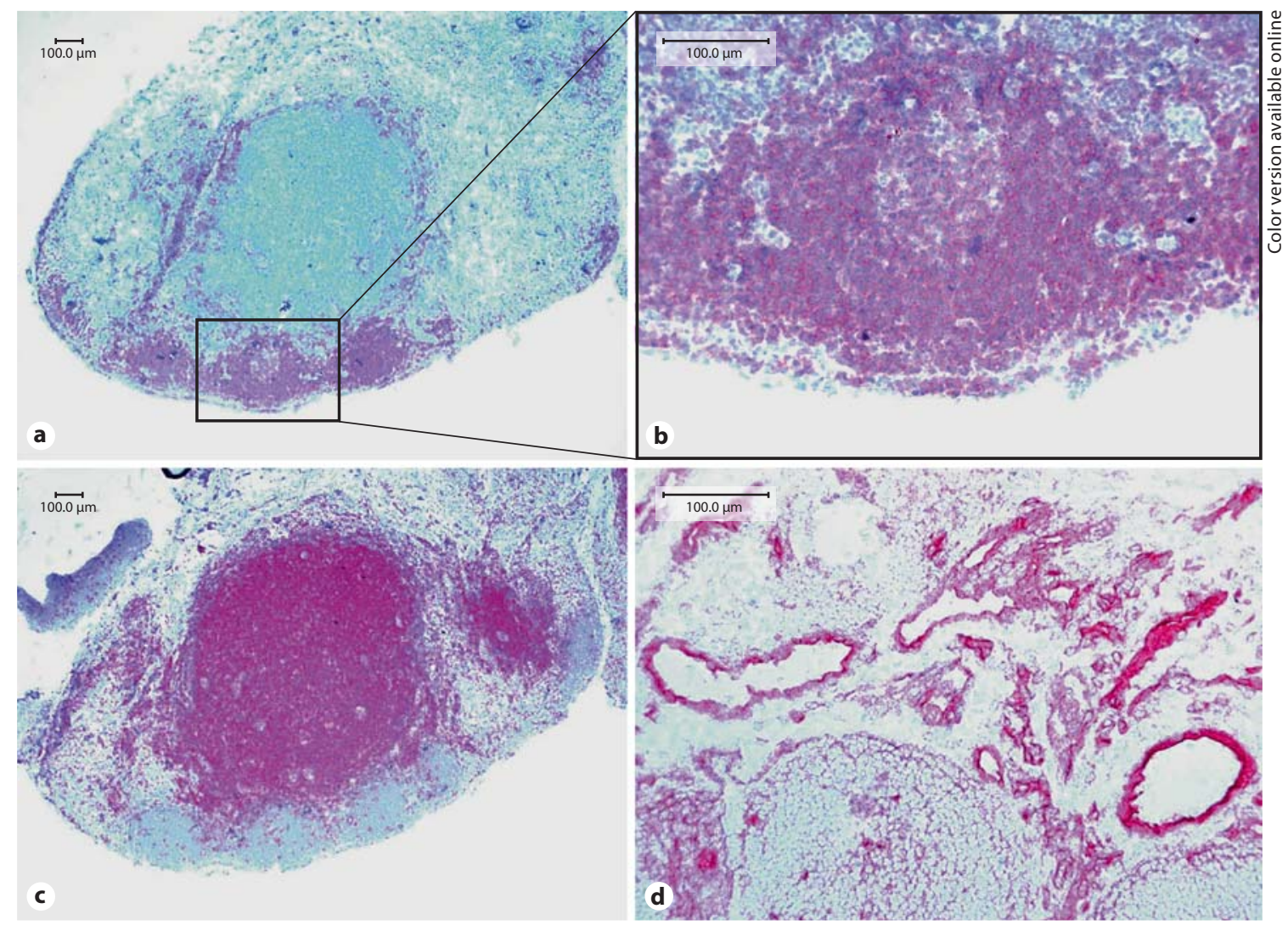

Fig. 4. Regenerated lymph node fragment 4 weeks after autologous transplantation and 1 week after PRP injection (group D). Cryosections were analyzed by immunohistological staining against B lymphocytes (a, b), T lymphocytes (c) and HEV RECA-1 marker (d).

\section{Discussion}

Understanding lymphangiogenesis and lymph node organogenesis can provide important insights into the mechanisms of survival of avascular lymph node transplants [15-17]. Transplants must undergo regenerative processes as the diffusion of nutrients through the lymph node capsule is not sufficient to maintain its avascular viability. This was observed after lymph node implantation underneath the kidney capsule [18] and in the mesentery in male rodents [19].

A difficulty encountered in our experiments was the dislocation of the transplants. This phenomenon accounts for the high number of empty probes. At many operation sites, more than 1 probe was taken because the area of the pouch seemed devoid of lymph nodes. Nevertheless, in 2 cases, viable lymph nodes were found in the pouch area, although they were registered as macroscopically invisible. Therefore, it seems that probes obtained for histological analysis are representative as they correspond to a random sample of the total.

A possible solution for this major problem would be to sew the transplanted lymph nodes to the underlying tissue. This technique was not primarily implemented because local lymph node reaction to the stitches was feared. However, it is likely that fixation of the transplants would optimize embedding and enhance regeneration. Alternatives like the use of tissue glue will be used in future experiments.

Lymph node fragmentation offers the possibility of multiplying regeneration foci without harvesting a high number of donor nodes. However, regeneration patterns suggest that whole lymph node transplants survive better than fragmented ones (fig. 6). The integrity of the capsule might be of relevance in this process, although reducing the size of the fragments would probably facilitate diffusion of nutrients. The fact that few whole lymph node transplants but almost half of the fragmented lymph 

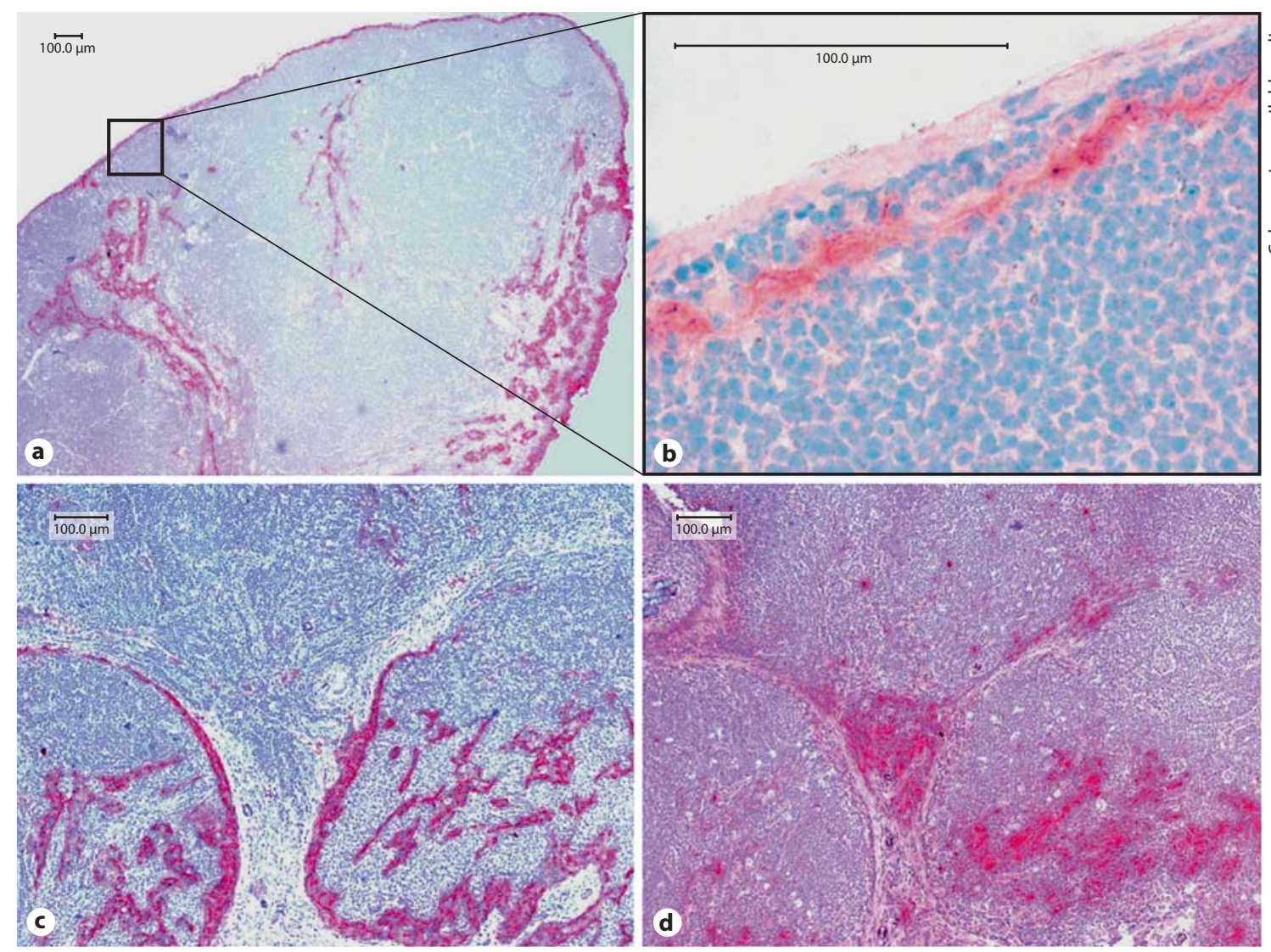

Fig. 5. Regenerated lymph node fragments 4 weeks after autologous transplantation and 1 week after PRP injection (group D). Cryosections were analyzed by immunohistological staining against LYVE-1 (a-c) and staining against Prox1 (d). LYVE-1 is only expressed in the lumen of lymphatics which explains the staining of the subcapsular sinus (b) and medullary sinuses (c). Prox1 is a specific marker for lymphatic endothelial cells with or without vessel maturation. This aspect explains the pattern observed in $\mathbf{d}$, where the central area is not producing LYVE-1, but Proxl staining lymphatic endothelial cells is present.
Fig. 6. Histogram showing the percentage of operative sites presenting regenerated, necrotic and combined regenerated and necrotic lymph nodes ( $\mathrm{LN}$ ) at a total of 37 operation sites submitted to histological examination 1 month after operation. In group $\mathrm{A}$ $(\mathrm{n}=11)$, whole lymph nodes were transplanted, and in groups $\mathrm{B}$ $(\mathrm{n}=11), \mathrm{C}(\mathrm{n}=8)$ and $\mathrm{D}(\mathrm{n}=7)$, fragmented lymph nodes were transplanted. In group C, sheep erythrocytes were injected intradermally 3 weeks after the operation, and in group D, PRP was injected.

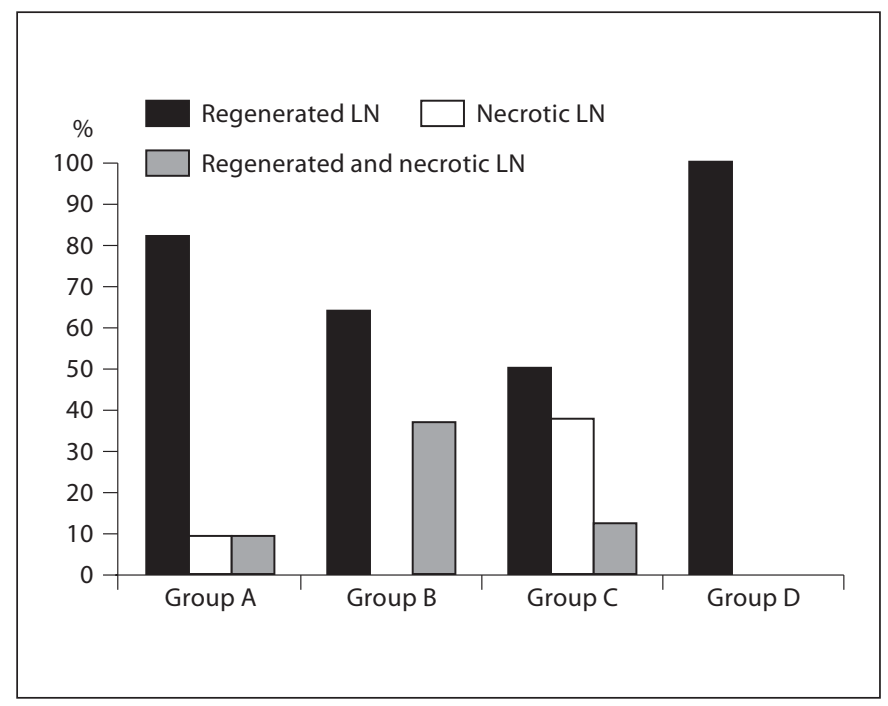


nodes in groups $\mathrm{B}$ and $\mathrm{C}$ were necrotic is probably due to the importance of 3-dimensional organization in the maintenance of lymph node viability [20].

Stimulation with sheep red blood cells as a particulate antigen has been proven to stimulate follicular development in the lymph nodes of other species [5]. However, peripheral injection of these antigens in the rat neither increased the number of germinal follicles in control group F, nor improved lymph node fragment regeneration in group C. Therefore, although sheep erythrocytes have clear antigenic potential in other species, they do not seem to induce a strong immunological reaction in the rat.

On the other hand, PRP not only improves the survival rate of transplanted lymph node fragments but also causes a B-cell proliferation in the lymph nodes of control group G. Platelets can be seen as pools of growth factors. Their $\alpha$-granules contain platelet-derived growth factor, transforming growth factor- $\beta$, plateletderived epidermal growth factor, insulin growth factor, platelet-derived angiogenesis factor, and growth factors of the vascular endothelial growth factor family, some of the most potent angiogenic and lymphangiogenic growth factors known [21-23]. However, their quantity can only be individually ascertained for each probe, a fact that renders this procedure difficult to reproduce. Not only can the composition of $\alpha$-granules vary in the same individual, but platelet counts in peripheral blood are also variable for each person. Additionally, differences between species should also be taken into account when interpreting experiments in animal models, as rat platelets contain less growth factors per platelet than human platelets [23]. However, PRP still possesses many advantages to outweigh its measurement difficulties, the first being that it is an autologous material. This and the fact that it is inexpensive and easy to isolate through simple centrifugation are clear advantages for its use in patients.
How the combination of platelet growth factors improves the regeneration of lymph node fragments probably depends on synergetic pathways leading to lymphangiogenesis. New blood vessels and lymphatics can profit from stimulation through growth factors contained in platelets, proven to promote maturation, and therefore, contributing to the improved stability of connections between the fragment and the surrounding tissues [16].

Our results showing that transplanted rat lymph node fragments are able to induce lymphatic and vascular proliferation and regain the function of the filtering peripheral lymph are consistent with concomitant research in the mouse [9]. Tammela et al. [9] very recently showed that fragmented lymph nodes transplanted in the axillary region of the mouse without surgical anastomosis were able to regenerate. After administration of a viral vector containing plasmids for vascular endothelial growth factor C, a significant improvement in lymphatic regeneration was described.

In conclusion, the results in this Lewis rat model strongly support the concept that the combination of surgical transplantation and the administration of syngeneic growth factors constitutes a possible solution for regeneration of new lymphatic vessels and a possible prevention of postoperative lymphedema. Experiments are now being undertaken to assess whether sequential repetitive doses further improve the results observed with a single injection of PRP.

\section{Acknowledgments}

This work was supported by a grant from the Jürgen Manchot Foundation, Düsseldorf. We thank Hugo Domingos for his valuable comments and Sheila Fryk for polishing the English. Andrea Herden and Karin Westermann provided excellent technical assistance.

\section{References}

1 Levick JR: Capillary filtration-absorption balance reconsidered in light of dynamic extravascular factors. Exp Physiol 1991;76: 825-857.

-2 Lämmermann T, Sixt M: The microanatomy of T-cell responses. Immunol Rev 2008;221: 26-43.

3 Williams AF, Franks PJ, Moffatt CJ: Lymphoedema: estimating the size of the problem. Palliat Med 2005;19:300-313.
4 Moffatt CJ, Franks PJ, Doherty DC, Williams AF, Badger C, Jeffs E, Bosanquet N, Mortimer PS: Lymphoedema: an underestimated health problem. QJM 2003;96:731738.

5 Pabst R, Rothkotter HJ: Regeneration of autotransplanted lymph node fragments. Cell Tissue Res 1988;251:597-601.
6 Blum KS, Radtke C, Knapp WH, Pabst R, Gratz KF: SPECT-CT: a valuable method to document the regeneration of lymphatics and autotransplanted lymph node fragments. Eur J Nucl Med Mol Imaging 2007;34: 1861-1867.

7 Pabst R, Westermann J, Rothkotter HJ: Immunoarchitecture of regenerated splenic and lymph node transplants. Int Rev Cytol 1991;128:215-260. 
8 Rothkotter HJ, Pabst R: Autotransplantation of lymph node fragments. Structure and function of regenerated tissue. Scand J Plast Reconstr Surg Hand Surg 1990;24:101-105.

$\checkmark 9$ Tammela T, Saaristo A, Holopainen T, Lyytikka J, Kotronen A, Pitkonen M, Abo-Ramadan U, Yla-Herttuala S, Petrova TV, Alitalo K: Therapeutic differentiation and maturation of lymphatic vessels after lymph node dissection and transplantation. Nat Med 2007;13:1458-1466.

- 10 Han J, Meng HX, Tang JM, Li SL, Tang Y, Chen ZB: The effect of different platelet-rich plasma concentrations on proliferation and differentiation of human periodontal ligament cells in vitro. Cell Prolif 2007;40:241252.

-11 Ogino Y, Ayukawa Y, Tsukiyama Y, Koyano $\mathrm{K}$ : The effect of platelet-rich plasma on the cellular response of rat bone marrow cells in vitro. Oral Surg Oral Med Oral Pathol Oral Radiol Endod 2005;100:302-307.

-12 Yazawa M, Ogata H, Kimura A, Nakajima T, Mori T, Watanabe N: Basic studies on the bone formation ability by platelet rich plasma in rabbits. J Craniofac Surg 2004;15:439446 .
13 Jila A, Kim H, Nguyen VP, Dumont DJ, Semple J, Armstrong D, Seto E, Johnston M: Lymphangiogenesis following obstruction of large postnodal lymphatics in sheep. Microvasc Res 2007;73:214-223.

14 Mäkinen T, Norrmen C, Petrova TV: Molecular mechanisms of lymphatic vascular development. Cell Mol Life Sci 2007;64:19151929.

15 Alitalo K, Tammela T, Petrova TV: Lymphangiogenesis in development and human disease. Nature 2005;438:946-953.

16 Aloisi F, Pujol-Borrell R: Lymphoid neogenesis in chronic inflammatory diseases. Nat Rev Immunol 2006;6:205-217.

17 Mebius RE: Lymphoid organogenesis: educating stroma. Immunol Cell Biol 2007;85 79-80.

18 Sasaki K, Pabst R, Rothkotter HJ: Development of the high endothelial venule in rat lymph node autografts. Anat Rec 1994;238: 473-479.

19 Ahrendt M, Hammerschmidt SI, Pabst O, Pabst R, Bode U: Stromal cells confer lymph node-specific properties by shaping a unique microenvironment influencing local immune responses. J Immunol 2008;181:18981907.
20 Ma B, Jablonska J, Lindenmaier W, Dittmar KE: Immunohistochemical study of the reticular and vascular network of mouse lymph node using vibratome sections. Acta Histochem 2007;109:15-28.

21 Boyapati L, Wang HL: The role of plateletrich plasma in sinus augmentation: a critical review. Implant Dent 2006;15:160-170.

$>22$ Stacker SA, Farnsworth RH, Karnezis T, Shayan R, Smith DP, Paavonen K, Davydova $\mathrm{N}$, Caesar C, Inder R, Baldwin ME, McColl BK, Roufail S, Williams RA, Hughes RA, Alitalo K, Achen MG: Molecular pathways for lymphangiogenesis and their role in human disease. Novartis Found Symp 2007; 281:38-43; discussion 44-53, 208-209.

23 van den Dolder J, Mooren R, Vloon AP, Stoelinga PJ, Jansen JA: Platelet-rich plasma: quantification of growth factor levels and the effect on growth and differentiation of rat bone marrow cells. Tissue Eng 2006;12: 3067-3073. 